\title{
'Listening to members is a large part of communicating effectively with them'
}

\author{
Helen Cobley reflects on her career and a decade as Executive General \\ Manager for the British Society of Periodontology (BSP).
}

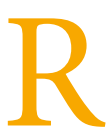

eflecting on ten years as Executive General Manager for the BSP inevitably led me to reflect on the whole of my career (43 years!) in and around general and dental health. The early years were spent at the clinical coalface, then in administration, both general and financial. At one point, rejoicing in the title of Assistant Director of Corporate Affairs, I was the Complaints Manager for a large Hospital Trust. The reason for mentioning this is that during my contemplation of what 43 years in health and dental care has taught me, communication and the effective use of language have been the recurrent watchwords leading to success or failure. The vast majority of complaints made by patients and their relatives are not about the care received, or not received, but about a failure of staff to communicate have not fully embraced social media. This is partly through fear of the unknown and partly because I prefer to read and write good English in proper sentence format. However, I do accept that social media is here to stay and it is the medium of choice for many people for receiving and sharing information. I still argue that language is key, however you chose to disseminate the message. The language we use in emails is fundamentally different to the language we used in the days of dictating and reviewing letters to individuals or groups. The 'Reply All' is a very dangerous button leading to red faces and indignation in equal measure! I have tried hard to instil an email code of practice. I have not succeeded as much as I had wished.

Listening to members is a large part of communicating effectively with them. The

\section{'Change is inevitable and should be embraced with enthusiasm and a constant awareness of the changes going on in the wider dental community...'}

effectively leading to misunderstanding and frustration as well as fear and a feeling of neglect. On a different stage, as a Magistrate, communication is key to the rule of law being applied fairly, proportionately and appropriately. Language is key!

What has this to do with the BSP? The same as it has with any organisation seeking to engage with its users and members. Targeted messaging, education and support has changed beyond all recognition in the last ten years and the need for clear, unequivocal communication has never been greater. I admit to being part dinosaur as I
BSP has listened and is, I believe, far more responsive to how we now interact with them. CPD and the manner in which the Society seeks to provide its members with education has changed in direct alignment with the technological developments of the last ten years. The offer of a two-hour session in a soulless hotel at the end of a long working day with only a curled up sandwich to sustain you lost its place in the CPD stakes with the arrival of the Webinar. What's not to like about a free-to-members two-hour vCPD session watchable at the time of the lecture or later if more convenient to you?

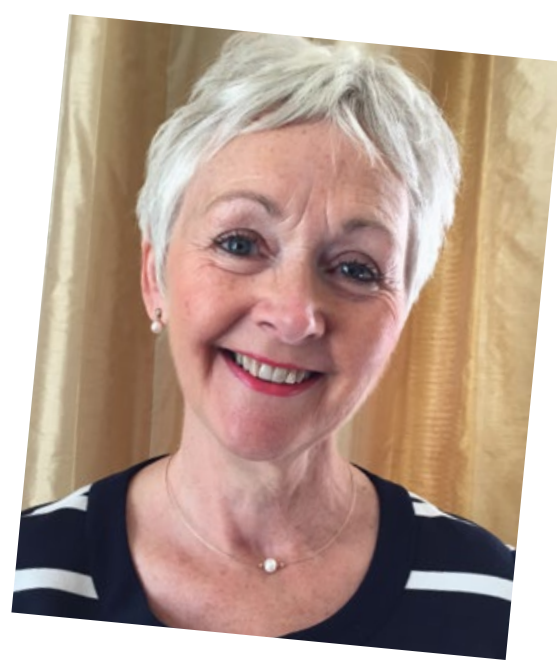

Conferences are beginning to take on a Marmite appearance: love them or loathe them. Can the same calibre of education be provided in another format? What would be lost by moving away from annual Big Top events to collaborative events with other organisations or smaller more regionally based events? The BSP is not alone in constantly juggling escalating costs with proportionate registration fees and a something-for-everyone programme. One thing I do know is that we have had some fantastic conferences with truly gratifying feedback and what do people really like about them? Networking, meeting up with old friends, listening live to world class speakers... in other words communicating orally and effectively on a personal level. Language is key - again.

Ten years is a long time in any sphere of medicine or dentistry. It is also true of periodontology as a specialism and in the context of the BSP. Entering its 70th year as the BSP, and registered as a Charity since 1974, I have been fortunate to be its EGM for the last ten years. These have been immensely challenging years and have seen the Society develop into a progressive and dynamic organisation with a growing membership - currently nudging 1,200 showing a 30\% increase over the decade - representing all members of the dental team, in general practice, hospital and academia. Change is inevitable and should be embraced, with caution maybe, but with enthusiasm and a constant awareness of the changes going on elsewhere in the wider dental community and healthcare provision as a whole. It is a new era for the BSP and I wish it well; may it continue to communicate effectively and well! 